\title{
Cystatin $C$ verbessert prognostische Aussage bei Niereninsuffizienz
}

\section{Die kombinierte Betrachtung von Serumkreatinin, des Albumin/Kreati- nin-Quotienten im Urin und Cystatin C verbessert die prognostische Aus- sage zur Mortalität und zum Risiko für ein terminales Nierenversagen.}

— Derzeit wird die chronische Niereninsuffizienz vorwiegend über die glomeruläre Filtrationsrate von unter $60 \mathrm{ml} /$ $\min / 1,73 \mathrm{~m}^{2}$ oder über einen Albumin/ Kreatinin-Quotienten (ACR) von 30 $\mathrm{mg} / \mathrm{g}$ oder mehr definiert. Beide Messungen sind durchaus problematisch.

Als neueren Parameter für den Grad der Nierenschädigung hat man in den letzten Jahren das Cystatin C entdeckt. In einer großen Populationsstudie an über 26000 US-Amerikanern im Durchschnittsalter von 65 Jahren wurden zwischen 2003 und 2010 die kombinierten Werte von die GFR, ACR und Cystatin $C$ bezüglich der prognostischen Bedeutung für die Gesamtmortalität und des Auftretens eines terminalen Nierenversagens untersucht. Von 26643 Teilnehmern starben in einem medianen Follow-up von 4,6 Jahren 1940. 177 entwickelten ein terminales Nierenversagen.
Im Vergleich zur Gruppe von Personen mit einer nur durch das Serumkreatinin definierten chronischen Niereninsuffizienz hatten Personen mit Niereninsuffizienz aufgrund der kombinierte Bewertung von Kreatinin und ACR eine Hazard Ratio für Tod von 3,3. Wurden alle drei Biomarker GFR, ACR und Cystatin C für die Definition des chronischen Nierenversagens herangezogen, so steigerte sich die Hazard Ratio sogar auf 5,6. Umgekehrt betrachtet wiesen $16 \%$ der Personen ohne chronische Niereninsuffizienz auf der Basis der GFR diese Erkrankung auf, wenn man ACR oder Cystatin C zusätzlich mit heranzog. Auch das Risiko für ein terminales Nierenversagen konnte durch die Betrachtung aller drei Parameter wesentlich besser vorhergesagt werden als bei alleiniger Berücksichtigung des Serumkreatinins.

\section{- C. A. Peralta et al.}

Detection of chronic kidney disease with creatinine, cystatin C, and Urine Albumin-to-Creatinine Ration and Association with Progression to End-Stage Renal Disease and Mortality. JAMA 306 (2011) 15, 1545-1552

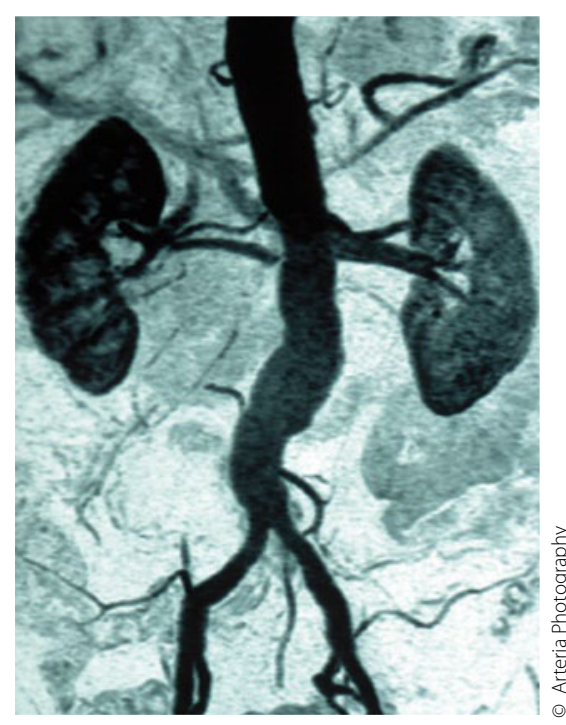

Wie lange halten diese Nieren noch durch?

\section{Kommentar}

Wie bei vielen Studien aus den USA ist eine Übertragung der Ergebnisse auf europäische Verhältnisse nicht ohne weiteres möglich, da 40\% der untersuchten Population afroamerikanischen Ursprungs waren. Die verbesserte Todesvorhersage erscheint für die ärztliche Praxis allerdings auch weniger von Relevanz. Die Wahrscheinlichkeit des Auftretens einer terminalen Niereninsuffizienz und damit der Notwendigkeit einer Dialysebehandlung ist jedoch sowohl individuell als auch versorgungspolitisch von größter Bedeutung.

H. S. FüESSL 